\title{
Influence on Calculated Blood Pressure of Measurement Posture for the Development of Wearable Vital Sign Sensors
}

\author{
Shouhei Koyama, ${ }^{1}$ Hiroaki Ishizawa, ${ }^{1}$ Akio Sakaguchi, ${ }^{2}$ \\ Satoshi Hosoya, ${ }^{2}$ and Takashi Kawamura ${ }^{2}$ \\ ${ }^{1}$ Institute for Fiber Engineering, Shinshu University, 3-15-1 Tokida, Ueda, Nagano, Japan \\ ${ }^{2}$ Faculty of Textile Science and Technology, Shinshu University, 3-15-1 Tokida, Ueda, Nagano, Japan \\ Correspondence should be addressed to Shouhei Koyama; shouhei@shinshu-u.ac.jp
}

Received 5 January 2017; Accepted 19 June 2017; Published 24 July 2017

Academic Editor: Christos Riziotis

Copyright (C) 2017 Shouhei Koyama et al. This is an open access article distributed under the Creative Commons Attribution License, which permits unrestricted use, distribution, and reproduction in any medium, provided the original work is properly cited.

\begin{abstract}
We studied a wearable blood pressure sensor using a fiber Bragg grating (FBG) sensor, which is a highly accurate strain sensor. This sensor is installed at the pulsation point of the human body to measure the pulse wave signal. A calibration curve is built that calculates the blood pressure by multivariate analysis using the pulse wave signal and a reference blood pressure measurement. However, if the measurement height of the FBG sensor is different from the reference measurement height, an error is included in the reference blood pressure. We verified the accuracy of the blood pressure calculation with respect to the measurement height difference and the posture of the subject. As the difference between the measurement height of the FBG sensor and the reference blood pressure measurement increased, the accuracy of the blood pressure calculation decreased. When the measurement height was identical and only posture was changed, good accuracy was achieved. In addition, when calibration curves were built using data measured in multiple postures, the blood pressure of each posture could be calculated from a single calibration curve. This will allow miniaturization of the necessary electronics of the sensor system, which is important for a wearable sensor.
\end{abstract}

\section{Introduction}

The "vital signs" include the blood pressure, heart rate, body temperature, respiratory condition, and consciousness level of a patient and can change according to physical and mental condition. Therefore, it is important to measure continuously and measure the variation of vital signs [1]. The elderly population in Japan continues to increase; soaring medical costs and lack of medical professionals are a serious issue [2]. Therefore, there is a need for a simple vital signs measurement system for elderly home health monitoring.

We have researched the development of a multi-vital sign sensor using a fiber Bragg grating (FBG) sensor, which is an optical-fiber-type highly accurate strain sensor [3-5]. FBG sensors have high sensitivity and accuracy and are compact and lightweight. They are resistant to adverse environmental and electromagnetic conditions, and multiple sensors can be connected in series to optical fibers. However, their manufacture from quartz glass renders them fragile; they require a large interrogator unit and a dedicated fiber-connector.

FBG sensors are mainly used in civil engineering and construction related fields. Tam et al. or Wei et al. presented fiber Bragg grating sensors for structural and railway applications [6-8]. In addition, vital signs measurement using the FBG has been previously reported [9-11]. The use of an optical fiber leads to a wearable sensor that is suitably compact, flexible, and lightweight. We suggested that weak pulse pressure changes could be measured by affixing the FBG sensor to the pulsation point of the skin surface with medical tape. A pulse wave signal is a change in the amount of blood flowing from the heart, and this change corresponds to the motion of the heart. Pressure from the blood flow is applied to the blood vessel; the pulsation point is a point where the strain from this pressure is propagated to the body surface. The strain change signal measured by the FBG sensor at the pulsation point includes blood pressure information. Pulse rate, respiration rate, and stress load can be measured from the pulse wave 


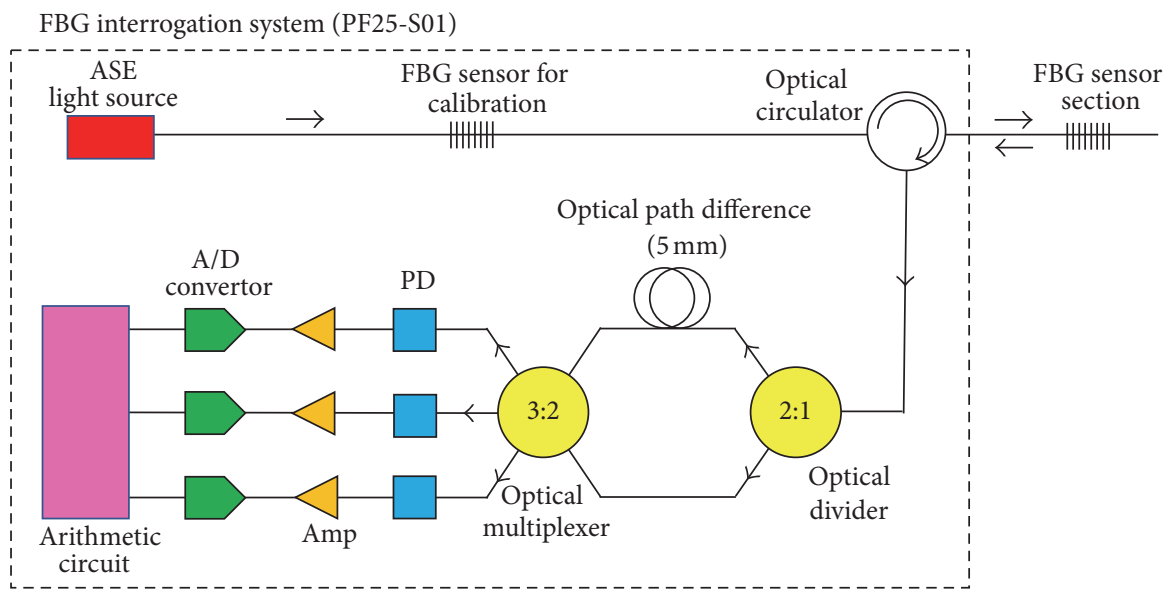

FIGURE 1: Schematic of the FBG interrogator.

signal measured with this FBG sensor [3]. Furthermore, since the pulse transit time (PTT) of the arrival time difference of the two waveforms is in a linear relationship with the systolic blood pressure $[12,13]$, systolic blood pressure was calculated by fixing the FBG sensor to two different points on the body surface [4].

Our ultimate goal is to develop a smart textile that is wearable and able to measure multiple vital signs. The optical fiber part of a FBG sensor can be easily introduced into textile products. In order to develop a wearable sensor using FBG sensor, it is necessary to reduce the size of the FBG interrogator. An arithmetic circuit is necessary, because a calibration curve is used in the proposed blood pressure calculation method. However, as the posture of the subject changes, the waveform of the pulse wave signal also changes, and the measurement error of the blood pressure calculated from the calibration curve is affected. In order to reduce this error, calibration curves corresponding to the posture can be constructed. However, multiple calibration curves require more complex calculations, leading to difficulties miniaturizing the arithmetic circuit, and thus wearable sensors cannot be developed. Therefore, it is necessary to have a calibration curve for calculating blood pressure that can reduce measurement error in various postures. In order to develop this calibration curve, the measurement posture of the subject and the reference blood pressure measurement method are verified. In addition, we discuss the issues related to the development of a wearable vital sign sensor using this method.

\section{Material and Methods}

2.1. FBG Sensor System. The FBG sensor is a device that periodically changes the refractive index along the axis of the core of the optical fiber to form a diffraction grating and reflects only a specific wavelength corresponding to the period $[14,15]$. Because the propagation direction of the light in the optical fiber is only in the traveling direction or the reflecting direction, the diffracted light in the FBG propagates only in the reflection direction. The wavelength
TABLE 1: Specifications of the FBG interrogator.

\begin{tabular}{lcc}
\hline Light source & \multicolumn{2}{c}{ ASE light source } \\
& $\begin{array}{c}\text { Output power } \\
\text { Wavelength range }\end{array}$ & $\begin{array}{c}30 \mathrm{~mW} \\
1525-1575 \mathrm{~nm}\end{array}$ \\
\hline \multirow{3}{*}{ Sensor part } & Length & $10 \mathrm{~mm}$ \\
& Resolution & $\pm 0.1 \mathrm{pm}$ \\
& Measurement range & $1550 \pm 0.5 \mathrm{~nm}$ \\
\hline \multirow{3}{*}{ Optical fiber } & Material & $\mathrm{SiO}_{2}$ \\
& Diameter & $145 \mathrm{~nm}$ \\
\hline Detector & Core diameter & $10.5 \mathrm{~nm}$ \\
\hline
\end{tabular}

of the reflected light from the FBG is called the Bragg wavelength:

$$
\lambda_{\text {Bragg }}=2 n_{\text {eff }} \Lambda
$$

where $\lambda_{\text {Bragg }}$ is the Bragg wavelength, $n_{\text {eff }}$ is the refractive index of the optical fiber core, and $\Lambda$ is the diffraction grating spacing. As the effective refractive index and diffraction grating interval, the Bragg wavelength also changes. Because the effective refractive index is constant during the measurement, the Bragg wavelength is determined only by the change of the diffraction grating interval. In other words, the FBG sensor functions as a sensor having sensitivity to strain caused by pressure applied to the diffraction grating portion.

The outline and specifications of the FBG sensor system using a Mach-Zehnder interferometer are shown in Figure 1 and Table 1. This interrogator detects a wavelength shift as an interference signal. Of the broadband near-infrared light from the light source, only the Bragg wavelength is reflected by the FBG sensor section, and the reflected light passes through the optical circulator and is transformed to an interference wave by the Mach-Zehnder interferometer. The light of the interference wave is divided into three directions, and the light intensity is detected by an indium gallium arsenide (InGaAs) photoelectric detector and an analog-todigital convertor (ADC). After demodulating the phase angle 


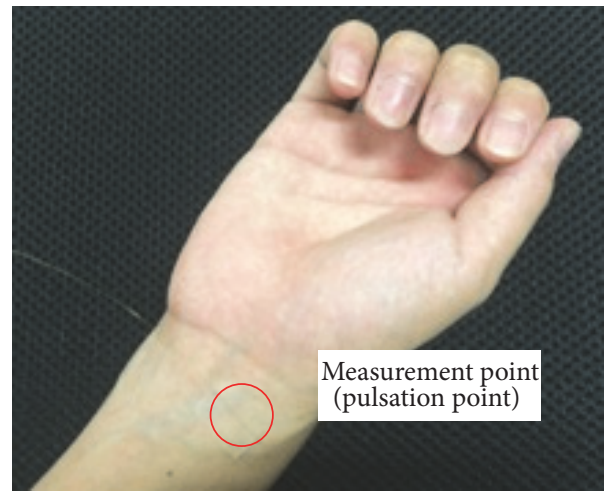

FIGURE 2: Experimental image by the FBG sensor measurement.

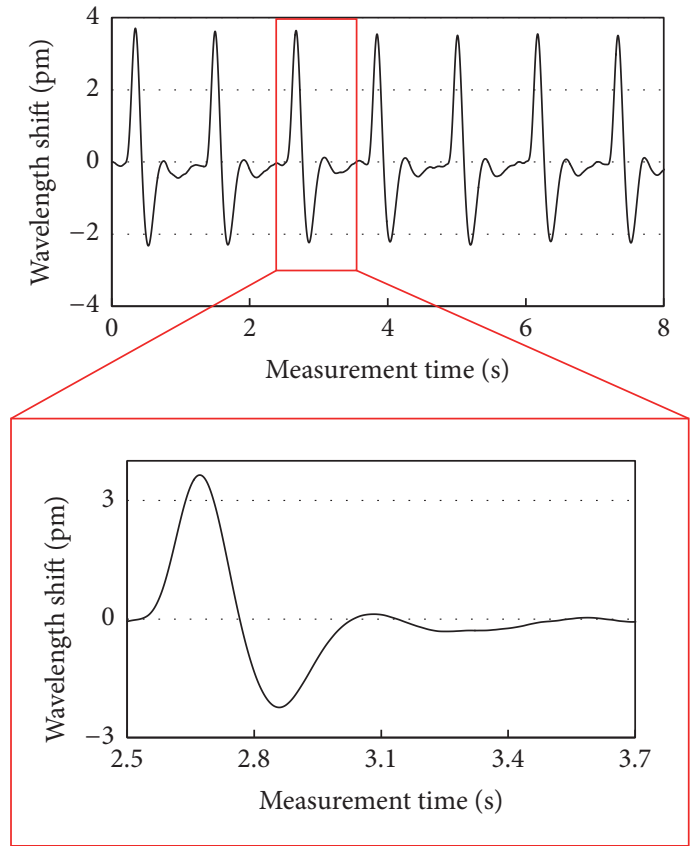

(a)

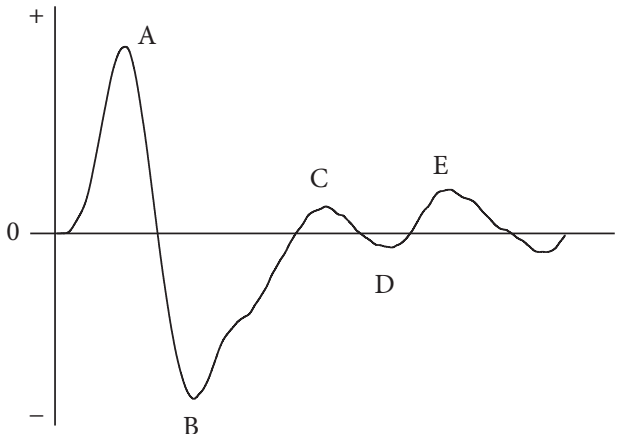

(b)

FIGURE 3: The pulse wave signals: (a) pulse wave signal measured by the FBG sensor; (b) pulse acceleration pulse wave of the second derivative of the pulse wave signal.

with a digital signal, wavelength shift (proportional to strain) is calculated $[5,16,17]$. With this detection method, the pressure applied to the FBG sensor is continuously measured.

\subsection{Measurement of the Pulse Wave Signal by the FBG Sensor.} The FBG sensor uses an optical fiber that reflects a wavelength of $1550 \mathrm{~nm}$. First, in order to measure the calibration signal, it is measured with the FBG sensor installed on the desk (no external force). Next, as per Figure 2, the FBG sensor is installed at the pulsation point of the subject's right wrist using medical tape, and the pulse wave signal is measured. We have already confirmed by basic experiment that the sensitivity of a FBG sensor does not change with medical tape or temperature. The pulse wave signal measured by the FBG sensor records the shift length of the Bragg wavelength from the calibration signal. The pulsation point is strained by the change in the vessel diameter caused by the force of the volume change of the artery propagating to the skin surface; thus, it contains blood pressure information. In other words, the pulse wave signal measured by the FBG sensor measures the change of the volume pulse wave signal.

Figure 3 shows the signal obtained by first differentiating the fundamental shape of human acceleration pulse wave (secondary differential signal of volume pulse wave) and the pulse wave signal measured by the FBG sensor [18]. The waveforms of these two pulse wave signals are similar. There are five characteristic peaks $\mathrm{A}$ to $\mathrm{E}$ in the acceleration pulse wave signal, which indicate the movement of the heart [19]. These peaks also appear in the pulse wave signal measured by the FBG sensor, and the blood pressure information 


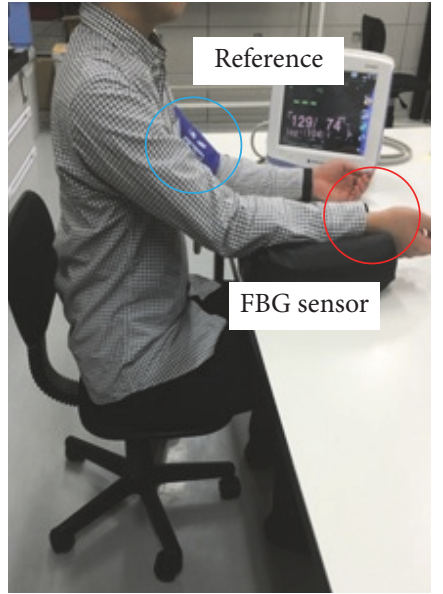

(a)

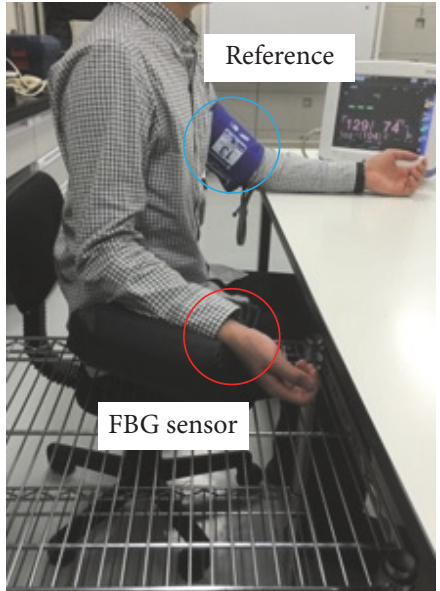

(b)

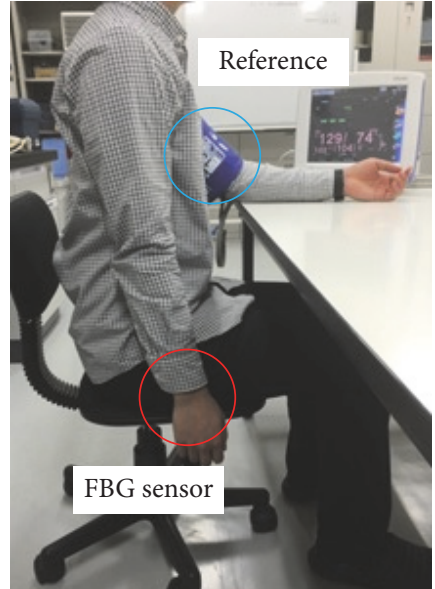

(c)

Figure 4: Three measurement postures in experiment 1: (a) $0 \mathrm{~cm}$ height difference (Position 1A), (b) $35 \mathrm{~cm}$ height difference (Position 1B), and (c) $70 \mathrm{~cm}$ height difference (Position 1C).

contained in these signals is calculated by PLS regression analysis.

The blood pressure is calculated from the pulse wave signal by the following method [5]. First, the pulse wave signal measured by the FBG sensor is processed by two signals, the first differential and a bandpass filter $(0.5-5 \mathrm{~Hz})$. Second, this signal is split at the peak position per pulse and averaged. Third, normalization processing (processed signal) is performed with the maximum point on the vertical axis of one divided pulse as "1" and the minimum point as " 0 ." It is analyzed using each reference blood pressure value measured simultaneously with this processed signal.

PLS regression is used in the analysis, which is a versatile multivariate analysis [20-23]. In PLS regression, the principal factor is calculated using the explanatory variable and the objective variable, and the regression equation is calculated with a new model using this factor. The difference between the new objective variable calculated from this regression equation and the first objective variable is compared. If the difference is significant, recalculate with the constructed model, calculate the second principal component factor, and verify it in the same way. Repeat this model building, calculate multiple principal component factors, and calculate a regression equation that reduces the error. The previous model when the difference is not significant is selected as the optimal model, and the regression equation is the calibration curve. In this paper, the signal processing pulse wave signal of the calibration data set is used as the explanatory variable, and the reference blood pressure value measured by the electronic sphygmomanometer is used as the objective variable. After the calibration curve is built, the blood pressure is calculated by substituting the signal processing pulse wave signal of the validation data set into this calibration curve. This calculated blood pressure and the reference blood pressure measured simultaneously with the substituted signal processing pulse wave signal are compared. From this result, the method of calculating the blood pressure with the FBG sensor will be verified.
2.3. Measurement Height and Measurement Posture. When calculating blood pressure from the pulse wave signal measured with the FBG sensor, the reference blood pressure value is important because PLS regression analysis is used. In addition, in order to use the FBG sensor as a wearable sensor in the future, the posture of the subject is important. In order to verify these two effects, it was measured under the following two experimental conditions.

When using the FBG sensor as a wearable sensor, the arm might be in different positions. In Experiment 1, the position of the wrist of the measuring part is changed while the subject is sitting, to investigate the influence due to the difference in height between the reference blood pressure measurement position and the FBG sensor. In Figure 4(a), the position of the right wrist where the FBG sensor is installed is the same height as the reference blood pressure measurement part (Position 1A). In addition, the pulse wave signal using the FBG sensor is measured for two other positions of the arm: lowered by $45^{\circ}$ (Position 1B) and lowered by $90^{\circ}$ (Position $1 C)$.

In Experiment 2, the subject's posture is changed with the reference blood pressure measurement value and the FBG sensor at the same height, in order to investigate the effect of posture without the influence of a measurement height difference. As shown in Figure 5, the pulse wave signal is measured with the subject in three postures: supine position, sitting position, and standing position. When measuring the pulse wave signal with the FBG sensor, reference blood pressure is simultaneously measured with an electronic sphygmomanometer on the left upper arm of the subject. The sampling frequency of the FBG interrogator is $10 \mathrm{kHz}$, and the measurement time is about 20 seconds. In Experiment 1, the pulse wave signal is measured 60 times; 50 data points are used to build the calibration curve and 10 data points for blood pressure calculation validation. In Experiment 2, the pulse wave signal is measured 100 times; 80 data points are used to build the calibration curve and 20 data points for blood pressure calculation validation. 


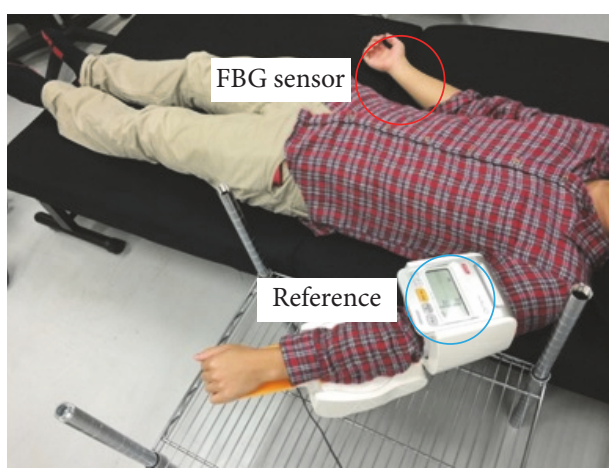

(a)

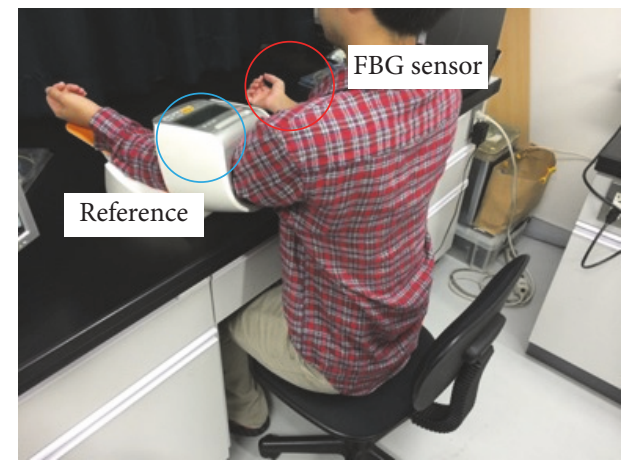

(b)

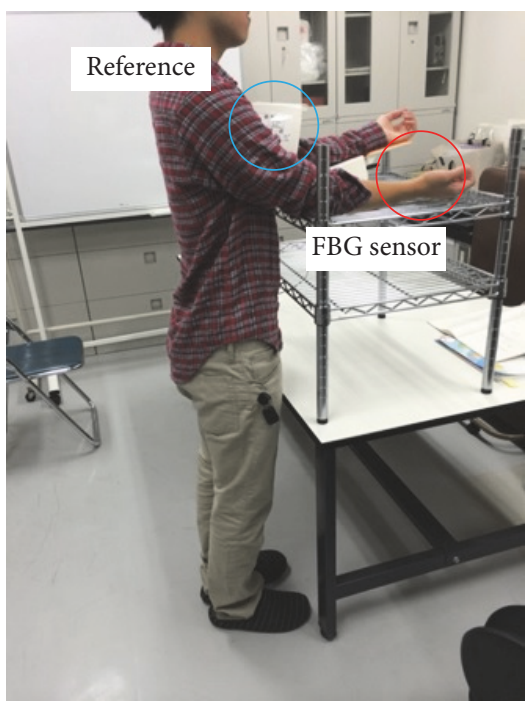

(c)

FIGURE 5: Three measurement postures in experiment 2: (a) supine position, (b) sitting position, and (c) standing position.

\section{Results and Discussion}

3.1. The Blood Pressure Calculation Result Based on the Difference of the Height of the Reference Blood Pressure Measurement Position and the FBG Pulse Wave Signal Measurement Position. Blood pressure was calculated from the pulse wave signal measured with the FBG measured in the states shown in Figure 4. The calibration data and validation data set for this experiment are shown in Table 2. The calibration curve was built by PLS regression analysis using this calibration data.

Table 3 shows the calibration curve results for each condition. Figure 6 shows the blood pressure results calculated from each calibration curve. For Position 1A the correlation coefficient of the calibration curve is high and the measurement error (standard error calibration, SEC) is as small as $3.0 \mathrm{mmHg}$. The measurement accuracy of the blood pressure calculated with this calibration curve (standard error of prediction, SEP) was $1.9 \mathrm{mmHg}$, which was a very good result. The measurement results when a height difference was introduced, Positions $1 \mathrm{~B}$ and $1 \mathrm{C}$, show that the SEP increases to $4.2 \mathrm{mmHg}$ and $5.7 \mathrm{mmHg}$, respectively. From this result, it was found that the calculated blood pressure is strongly
TABLE 2: Calibration data and validation data set in Experiment 1.

(a) Calibration data set

\begin{tabular}{|c|c|c|c|c|}
\hline & Samples & $\operatorname{Max}(\mathrm{mmHg})$ & Min. $(\mathrm{mmHg})$ & Avg. (mmHg) \\
\hline Position 1A & 50 & 121 & 103 & 111.3 \\
\hline Position $1 \mathrm{~B}$ & 50 & 119 & 99 & 108.8 \\
\hline Position 1C & 50 & 117 & 90 & 104.9 \\
\hline
\end{tabular}

(b) Validation data set

\begin{tabular}{|c|c|c|c|c|}
\hline & Samples & $\operatorname{Max}(\mathrm{mmHg})$ & Min. $(\mathrm{mmHg})$ & Avg. (mmHg) \\
\hline Position $1 \mathrm{~A}$ & 10 & 117 & 104 & 110.7 \\
\hline Position $1 \mathrm{~B}$ & 10 & 116 & 98 & 107.3 \\
\hline Position $1 \mathrm{C}$ & 10 & 116 & 90 & 103.8 \\
\hline
\end{tabular}

affected by the height difference between the reference blood pressure measurement position and the FBG sensor.

The blood pressure increases by about $7.8 \mathrm{mmHg}$ if the measurement height differs by $10 \mathrm{~cm}$. The difference in height of the measured position in this experiment is about $35 \mathrm{~cm}$ for Position $1 \mathrm{~B}$ and about $70 \mathrm{~cm}$ for that in Position 1C. Therefore, for the calculations, it contains errors of about 


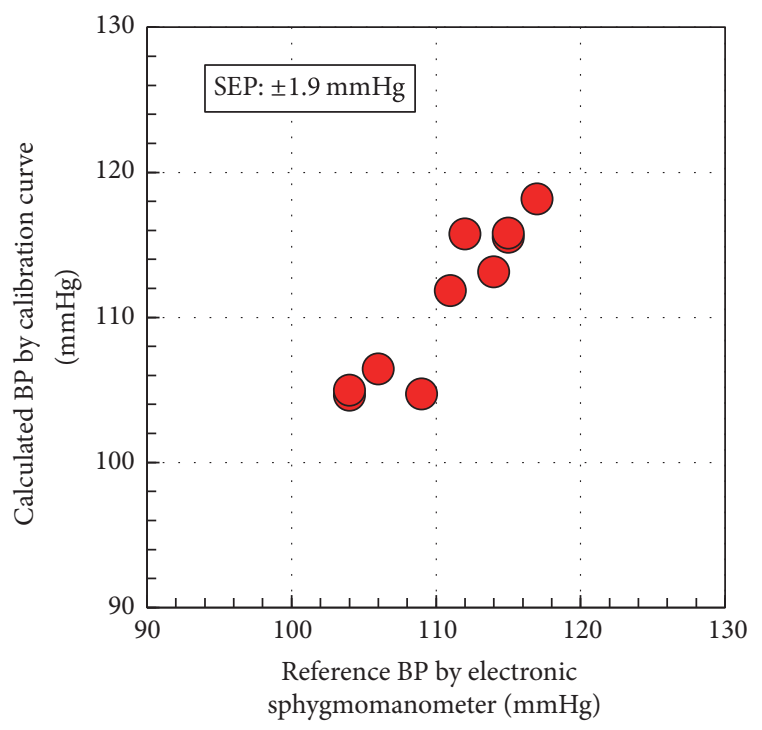

(a) Position $1 \mathrm{~A}, 0 \mathrm{~cm}$ difference

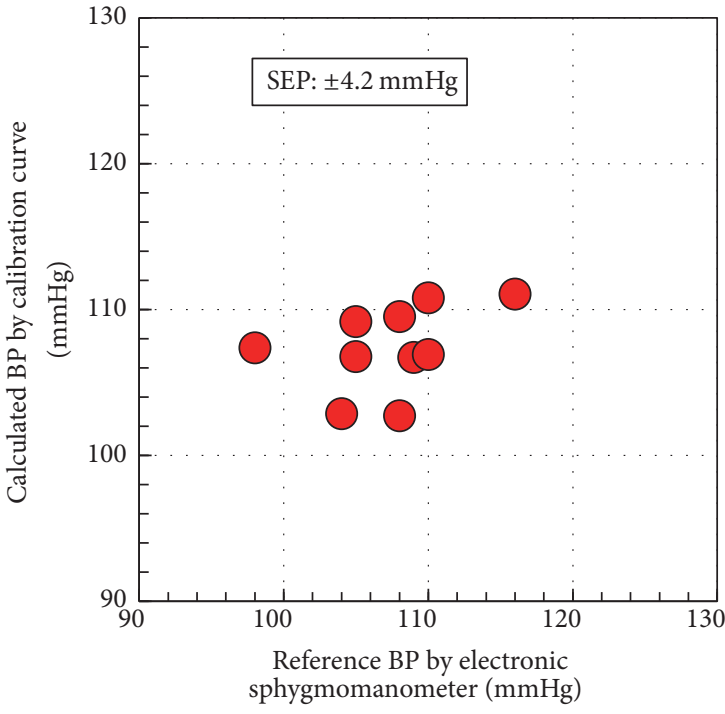

(b) Position 1B, $35 \mathrm{~cm}$ difference

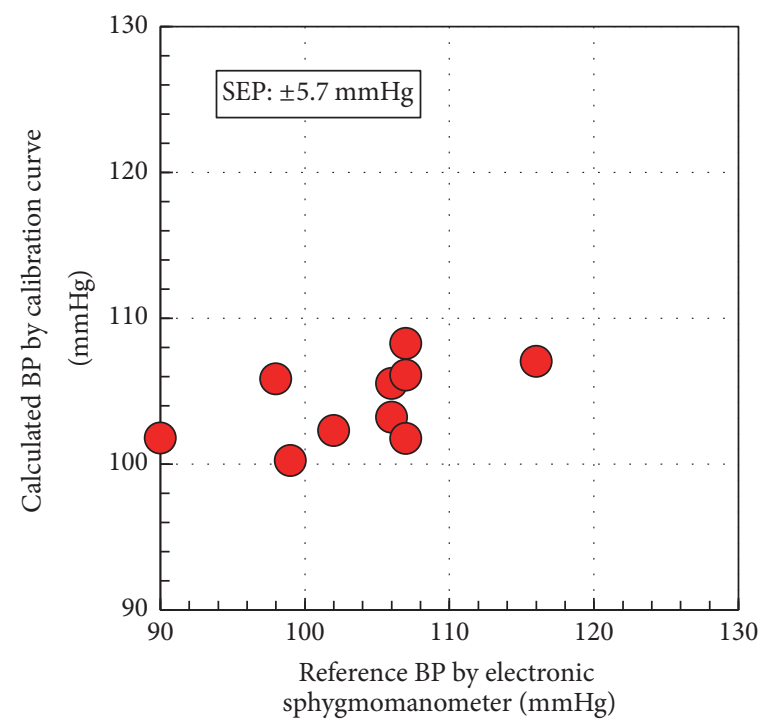

(c) Position 1C, $70 \mathrm{~cm}$ difference

Figure 6: The results of calculated blood pressure in each posture: (a) Position 1A, (b) Position 1B, and (c) Position 1C.

TABLE 3: Calibration curve results in Experiment 1.

\begin{tabular}{lccc}
\hline & Factors & Correlation coefficient & SEC $(\mathrm{mmHg})$ \\
\hline Position 1A & 4 & 0.84 & 3.0 \\
Position 1B & 4 & 0.27 & 4.4 \\
Position 1C & 4 & 0.13 & 5.7 \\
\hline
\end{tabular}

$27.2 \mathrm{mmHg}$ for $35 \mathrm{~cm}$ difference and about $54.4 \mathrm{mmHg}$ for $70 \mathrm{~cm}$ difference. This error is very large because the mean value of the reference blood pressure of the $35 \mathrm{~cm}$ difference is $108.8 \mathrm{mmHg}$ ( $25 \%$ relative to the mean blood pressure) and of the $70 \mathrm{~cm}$ difference is $104.9 \mathrm{mmHg}$ (52\% against the mean blood pressure). In other words, the calibration curves have been built by including large errors in the reference blood pressure. Therefore, the blood pressure calculated from these calibration curves has a large error. From the above results, the height of the measured position of the reference blood pressure is important when building the calibration curve, and should be measured at the same height as the FBG measurement position.

3.2. Blood Pressure Calculation Results for Different Measurement Postures. When building the calibration curve in Section 3.1, the reference blood pressure measurement and the FBG were measured at the same height. In this section, the dependence of the blood pressure calculation on the posture of the subject is investigated. The subjects are in supine, sitting, and standing positions, and the pulse wave signal is measured by the FBG sensor. The calibration data and validation data set in this experiment are shown in Table 4. Table 5 shows the calibration curve results for each 


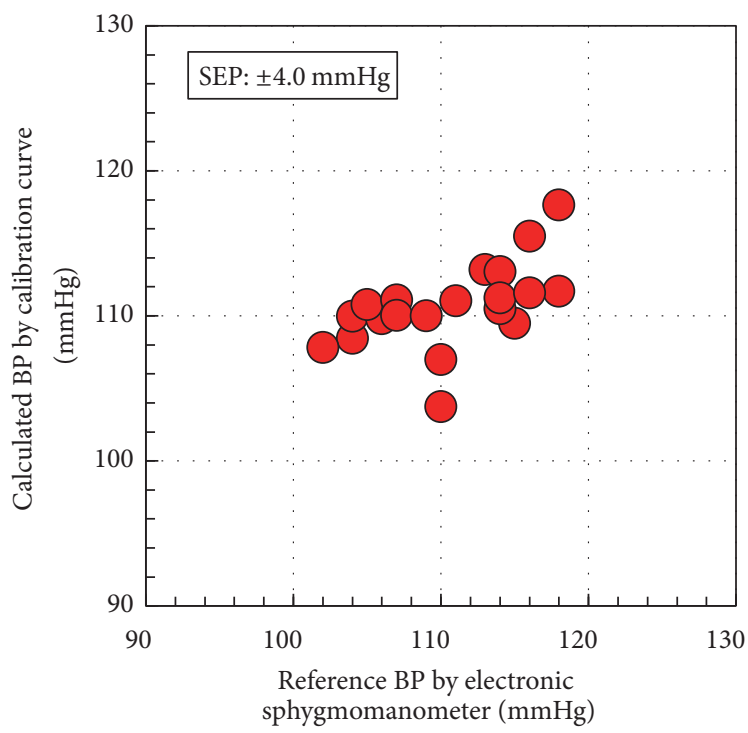

(a) Supine position

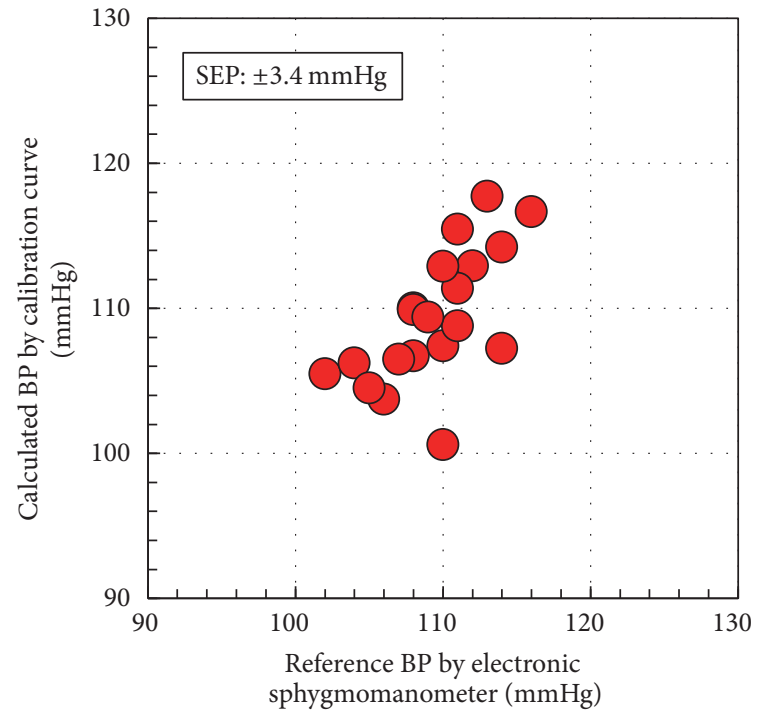

(b) Sitting position

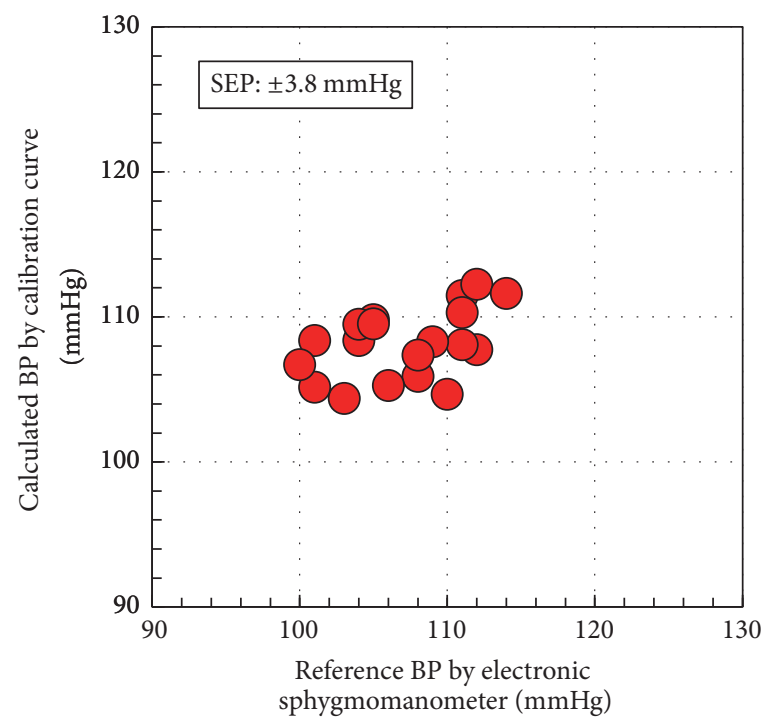

(c) Standing position

FIGURE 7: The results of calculated blood pressure in each posture: (a) supine position, (b) sitting position, and (c) standing position.

TABLE 4: Calibration data and validation data set in Experiment 2.

(a) Calibration data set

\begin{tabular}{lcccc}
\hline & Samples & Max $(\mathrm{mmHg})$ & Min. (mmHg) & Avg. (mmHg) \\
\hline Supine & 80 & 120 & 101 & 111.3 \\
Sitting & 80 & 121 & 99 & 110.9 \\
Standing & 80 & 118 & 100 & 107.6 \\
\hline
\end{tabular}

(b) Validation data set

Samples Max (mmHg) Min. (mmHg) Avg. (mmHg)

\begin{tabular}{lllll}
\hline Supine & 20 & 118 & 102 & 110.7 \\
Sitting & 20 & 116 & 102 & 109.5 \\
Standing & 20 & 114 & 100 & 107.1 \\
\hline
\end{tabular}

TABLE 5: Calibration curve results in Experiment 2.

\begin{tabular}{lccc}
\hline & Factors & Correlation coefficient & SEC $(\mathrm{mmHg})$ \\
\hline Supine & 4 & 0.69 & 3.5 \\
Sitting & 4 & 0.84 & 2.9 \\
Standing & 4 & 0.56 & 3.6 \\
\hline
\end{tabular}

condition. The results of blood pressure calculations for these postures are shown in Figure 7. These results show that the measurement accuracy of calculated blood pressure in each position was very good, at less than $4.0 \mathrm{mmHg}$. Compared with the results in Section 3.1, the measurement accuracy is improved. Therefore, when building a calibration curve, it is 
TABLE 6: Calibration data and validation data sets in all-posture experiment.

(a) Calibration data set

\begin{tabular}{lcccc}
\hline & Samples & Max $(\mathrm{mmHg})$ & Min. (mmHg) & Avg. (mmHg) \\
\hline All & 120 & 120 & 100 & 110.0 \\
(Supine) & 40 & 120 & 100 & 110.2 \\
(Sitting) & 40 & 119 & 101 & 109.7 \\
(Standing) & 40 & 119 & 102 & 110.0 \\
\hline
\end{tabular}

(b) Validation data set

\begin{tabular}{lcccc}
\hline & Samples & Max (mmHg) & Min. (mmHg) & Avg. (mmHg) \\
\hline All & 30 & 121 & 103 & 110.7 \\
(Supine) & 10 & 121 & 105 & 110.8 \\
(Sitting) & 10 & 116 & 104 & 110.1 \\
(Standing) & 10 & 119 & 103 & 111.2 \\
\hline
\end{tabular}

important to measure the reference blood pressure and the FBG pulse wave signal at the same height.

In order to develop a wearable FBG sensor, the blood pressure must be calculated in various postures. On the other hand, to selectively use calibration curves for each posture, multiple calibration curve programs are required, and blood pressure cannot be calculated except for the constructed posture. Therefore, in order to calculate blood pressure for various postures, a calibration curve is built using the data sets for three postures. 40 data points were selected from the supine, sitting, and standing positions calibration data sets, and a calibration curve (all-posture calibration curve) was constructed with the total of 120 data points. The calibration curve thus built is assigned 10 data points from each of the validation data sets of the three positions, and the blood pressure is calculated. The calibration data and validation data set in this experiment are shown in Table 6 . The blood pressure calculation result is shown in Figure 8. The correlation coefficient of the all-posture calibration curve is 0.69 , SEC is $3.8 \mathrm{mmHg}$, and the measurement accuracy of the calculated blood pressure is $3.2 \mathrm{mmHg}$.

The measurement accuracies for each posture, given in Table 7(b), were $3.0,2.8$, and $3.8 \mathrm{mmHg}$, for the supine, sitting, and standing postures, respectively. Figure 9 shows the factor loading of the calibration curve in Table 7(a). This calibration curve is built with 4 factors, so the number of factor loadings is 4 . The larger the absolute value of factor loading, the greater the effect of strain on that measurement time. The absolute value of factor loading 1 (blue line in Figure 9(a)) is large at measurement times 0 (arrow 1-a) and 0.25 (arrow 1-b). These measurement times are "Peak A" and "Between Peaks B and C" in Figure 9(b). Since Peak B in Figure 9(b) is normalized to "0," "Between Peaks B and C" shows the magnitude of "Peak C" against "Peak B". Similarly, all factor loading absolute values are large in "Peak A," "Between Peak B and C" and "Peak C" in Figure 9(b). In other words, this calibration curve reflects the influence of "Peaks $\mathrm{A}, \mathrm{B}$, and C" indicating systolic heart motion. Therefore, from

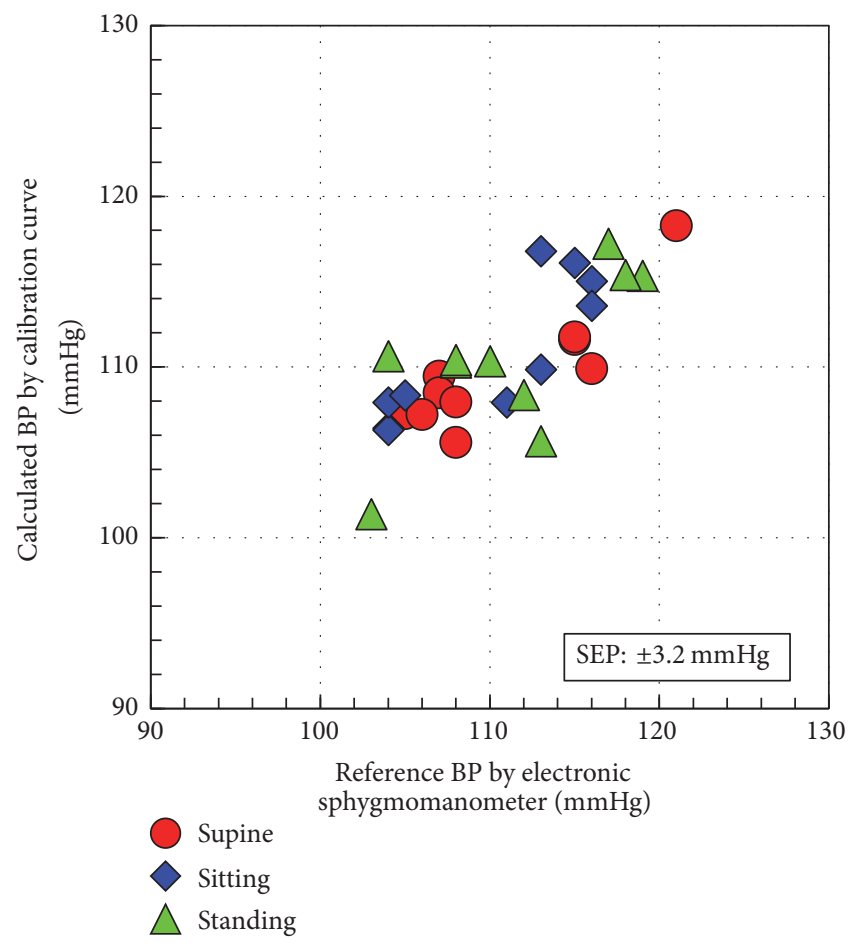

FIGURE 8: Results of calculated blood pressure by all-posture calibration curve.

TABLE 7: Calibration curve result and calculation blood pressure in each posture for all-posture calibration curve.

(a) All-posture calibration curve result

\begin{tabular}{lccc}
\hline & Factors & Correlation coefficient & SEC $(\mathrm{mmHg})$ \\
\hline All-posture & 4 & 0.69 & 3.8 \\
\hline
\end{tabular}

(b) Results of the calculation blood pressure in each posture

\begin{tabular}{lcccc}
\hline & All & Supine & Sitting & Standing \\
\hline SEP $(\mathrm{mmHg})$ & 3.2 & 3.0 & 2.8 & 3.8 \\
\hline
\end{tabular}

these factors loading, the calibration curve is built from the magnitude of strain by the pulse wave, and the blood pressure has been calculated from this calibration curve. These results show that highly accurate blood pressure is calculated in all postures, using a single calibration curve. The use of a single curve may aid the effectiveness and future miniaturization of a wearable device.

The above results show that if the FBG sensor pulse wave signal is measured at the same height as the reference blood pressure, the blood pressure can be calculated with high accuracy regardless of the posture. Furthermore, when data measured in a plurality of postures are used when building the calibration curve, the blood pressure at each posture is calculated with one calibration curve. In this experiment, the same tendency was also shown by calculating the diastolic blood pressure. Therefore, it is possible to calculate systolic and diastolic blood pressure simultaneously. 


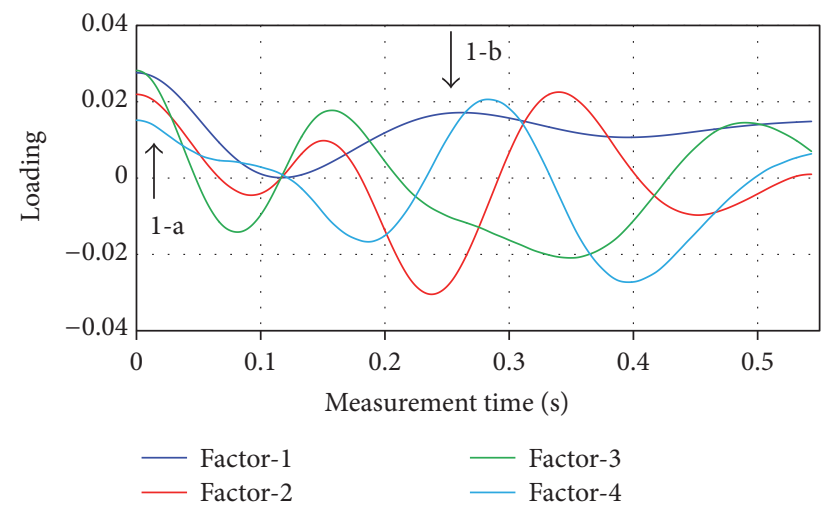

(a) Factor loading of the calibration curve

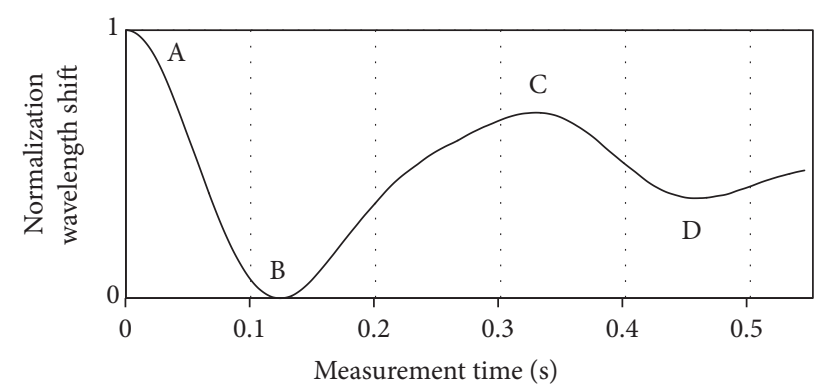

(b) Normalization single pulse wave signal

FIGURE 9: Results of factor lording of the calibration curve and Normalization single pulse wave signal.

\section{Conclusions}

In this paper, the influence of measurement height and posture on the method of calculating blood pressure from a calibration curve using pulse wave signals measured with FBG sensor was demonstrated. The findings obtained in this study are listed below.

(i) When the FBG sensor and the reference blood pressure were measured at the same height, the measurement accuracy of the calculated blood pressure was good.

(ii) When the heights are different, the measurement accuracy of the calculated blood pressure becomes worse as the height difference increases.

(iii) Even if the measurement posture is changed, if the height is the same, the measurement accuracy of the calculated blood pressure was good.

(iv) When using a calibration curve created using measurement data from multiple postures, the accuracy of blood pressure measurement calculated for all postures was good.

The results above show that the method of measuring the reference blood pressure value is important, and it is improved by measuring it at the same height as the FBG sensor. In this study, reference blood pressure was measured using an electronic sphygmomanometer on the upper arm. Considering the result of this study, it is better to measure the reference blood pressure at the wrist because the FBG sensor is installed on the wrist. Therefore, it is necessary to change the blood pressure monitor for measuring the reference blood pressure. Fortunately, since blood pressure monitors for measuring the wrist part are commercially available, it is possible to build a more accurate calibration curve. When the sphygmomanometer is installed on the left wrist and the FBG sensor is installed on the right wrist, the signal can be measured at the same measurement height even in the postures in which both arms are partially bent or completely lowered. Even if the posture is changed as in Section 3.2, because the calculated blood pressure measurement accuracy is good, accurate blood pressure will be calculated in these conditions as well. The accuracy of the potentially wearable device may be further improved by developing a calibration curve that includes data from many more possible postures. Therefore, this leads to cost going down when selling wearable sensor kit.

Miniaturization of the whole device is important for the development of a wearable sensor. We have already developed a method to introduce the sensing optical fiber into textile products [24]. Miniaturized electronics, light source, detector, and batteries from consumer electronics are readily available. Since it was shown that a simple calibration curve can be built from the results of this time, the problem of miniaturization of the arithmetic circuit will be solved. Thus, a compact sensor system using FBG sensors can be developed, and this system can be introduced into wearable textile products; these might include integration in the sleeves of clothes or wristband-type devices. The findings found on this paper are important for developing a wearable blood pressure sensor. In the near future, the authors intend to publish a study of developed wearable sensors using FBG sensors.

\section{Conflicts of Interest}

The authors declare that there are no conflicts of interest regarding the publication of this paper.

\section{Acknowledgments}

This work was supported by JSPS KAKENHI Grant no. JP16H01805 and the Wearable Vital Signs Measurement System Development Project at Shinshu University.

\section{References}

[1] H. S. Wolff, "Instruments for the investigation of the physiological and environmental patterns of normal life," in Proceedings of the In Digest of the 6th International Conference on Medical Electronics and Biological Engineering, pp. 309-312, Japanese Physiological Society, Tokyo, Japan, August 1965.

[2] Cabinet Office, "Government of Japan. Situation on Aging, Annual Report on the Aging Society 2015," 2016, http://www8 .cao.go.jp/kourei/english/annualreport/2015/pdf/cl-1.pdf.

[3] Y. Miyauchi, H. Ishizawa, and S. Koyama, "The pulse rate measuring system which use FBG sensors, light sources 2012," in Proceedings of the 13th International Symposium on the Science 
and Technology of Lighting, pp. 415-416, New York, NY, USA, June 2012.

[4] Y. Miyauchi, H. Ishizawa, S. Koyama, and S. Sato, "Verification of the systolic blood-pressure measurement principle by FBG sensors," in Proceedings of the SICE Annual Conference 2012 Final Program and Papers, pp. 1852-1854, Akita, Japan, August 2012.

[5] S. Koyama, H. Ishizawa, K. Fujimoto, S. Chino, and Y. Kobayashi, "Influence of Individual Differences on the Calculation Method for FBG-Type Blood Pressure Sensors," Sensors, vol. 17, no. 1, p. 48, 2017.

[6] H. Y. Tam, S. Y. Liu, B. O. Guan, W. H. Chung, T. H. T. Chan, and L. K. Cheng, "Fiber bragg grating sensors for structural and railway applications," in Proceedings of the Advanced Sensor Systems and Applications II, pp. 85-97, Beijing, China, November 2004.

[7] C. Wei, C. Lai, S. Liu et al., "A fiber bragg grating sensor system for train axle counting," IEEE Sensors Journal, vol. 10, no. 12, pp. 1905-1912, 2010.

[8] C. Wei, Q. Xin, W. H. Chung, S. Liu, H. Tam, and S. L. Ho, "Realtime train wheel condition monitoring by fiber bragg grating sensors," International Journal of Distributed Sensor Networks, vol. 2012, Article ID 409048, 7 pages, 2012.

[9] J. Hao, M. Jayachandran, P. L. Kng, S. F. Foo, P. W. Aung Aung, and Z. Cai, "FBG-based smart bed system for healthcare applications," Frontiers of Optoelectronics in China, vol. 3, no. 1, pp. 78-83, 2010.

[10] W. B. Spillman Jr., M. Mayer, J. Bennett et al., "A 'smart' bed for non-intrusive monitoring of patient physiological factors," Measurement Science and Technology, vol. 15, no. 8, pp. 16141620, 2004.

[11] T. Elsarnagawy, J. Haueisen, M. Farrag, S. G. Ansari, and H. Fouad, "Embedded fiber bragg grating based strain sensor as smart costume for vital signal sensing," Sensor Letters, vol. 12, no. 11, pp. 1669-1674, 2014.

[12] H. Gesche, D. Grosskurth, G. Küchler, and A. Patzak, "Continuous blood pressure measurement by using the pulse transit time: comparison to a cuff-based method," European Journal of Applied Physiology, vol. 112, no. 1, pp. 309-315, 2012.

[13] F. S. Cattivelli and H. Garudadri, "Noninvasive cuffless estimation of blood pressure from pulse arrival time and heart rate with adaptive calibration," in Proceedings of the 2009 6th International Workshop on Wearable and Implantable Body Sensor Networks, BSN 2009, pp. 114-119, Berkeley, Calif, USA, June 2009.

[14] K. O. Hill, Y. Fuji, C. D. Johnson, and B. S. Kawasaki, "Photosensitivity in optical fiber waveguide: application to reflection filters fabrication," Applied Physics Letters, vol. 32, no. 10, pp. 647-649, 1978.

[15] M. M. Werneck, R. C. S. B. Allil, B. A. Ribeiro, and F. V. B. de Nazaré, A Guide to Fiber Bragg Grating Sensors, Current Trends in Short- and Long-period Fiber Gratings, Intech, 978-953-511131-3, chapter 1, 2013.

[16] T. Yoshino, Y. Sano, D. Ota, K. Fujita, and T. Ikui, "Fiber-bragggrating based single axial mode fabry-perot interferometer and its strain and acceleration sensing applications," Journal of Lightwave Technology, vol. 34, no. 9, Article ID 7390182, pp. 2240-2250, 2016.

[17] M. D. Todd, G. A. Johnson, and C. C. Chang, "Passive, light intensity-independent interferometric method for fibre Bragg grating interrogation," Electronics Letters, vol. 35, no. 22, pp. 1970-1971, 1999.
[18] J. M. Ahn, "Wave detection in acceleration plethysmogram," Healthcare Informatics Research, vol. 21, no. 2, pp. 111-117, 2015.

[19] M. Elgendi, I. Norton, M. Brearley, D. Abbott, and D. Schuurmans, "Detection of a and b waves in the acceleration photoplethysmogram," BioMedical Engineering Online, vol. 13, no. 1, no. 139, pp. 139-158, 2014.

[20] H. Martens and T. Naes, Multivariate Calibration, John Wiley \& Sons, London, UK, 1989.

[21] S. Wold, A. Ruhe, H. Wold, and W. J. Dunn, "The collinearity problem in linear regression. The Partial Least Squares (PLS) approach to generalized inverses," SIAM Journal on Scientific and Statistical Computing, vol. 5, no. 3, pp. 735-743, 1984.

[22] S. Wold, M. Sjostrom, and L. Ericksson, "PLS-regression, a basic tool of chemometrics," ChemomIntell Lab Syst, vol. 58, pp. 109130, 2001.

[23] A. Lorber, L. E. Wangen, and B. R. Kowalski, "A theoretical foundation for the PLS algorithm," Journal of Chemometrics, vol. 1, no. 1, pp. 19-31, 1987.

[24] A. Sakaguchi, M. Kato, H. Ishizawa, H. Kimura, and S. Koyama, "Fabrication of optical fiber embedded knitted fabrics for smart textiles," Journal of Textile Engineering, vol. 62, no. 6, pp. 129$134,2016$. 


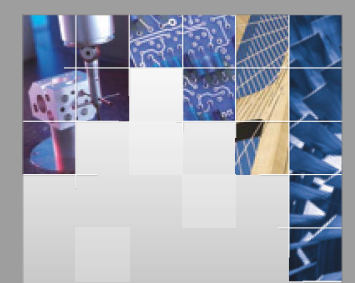

\section{Enfincering}
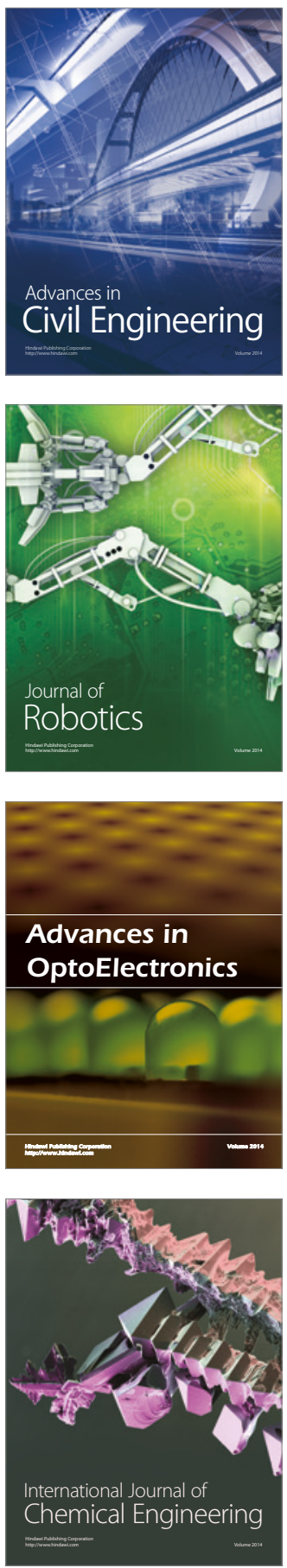

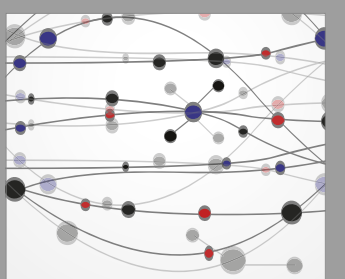

The Scientific World Journal

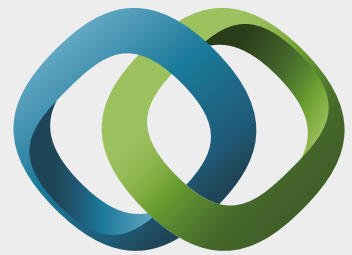

\section{Hindawi}

Submit your manuscripts at

https://www.hindawi.com
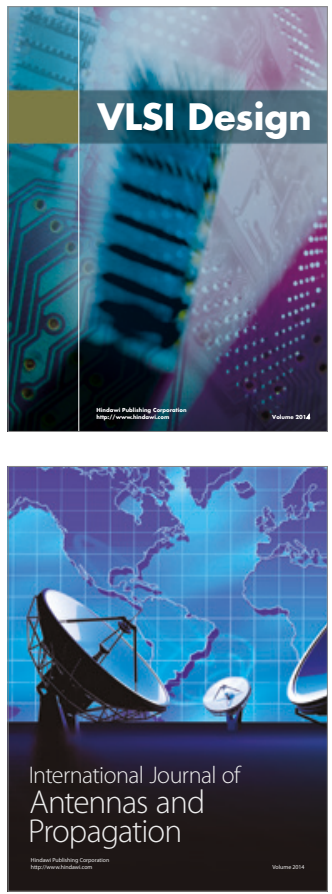

\section{Rotating}

Machinery
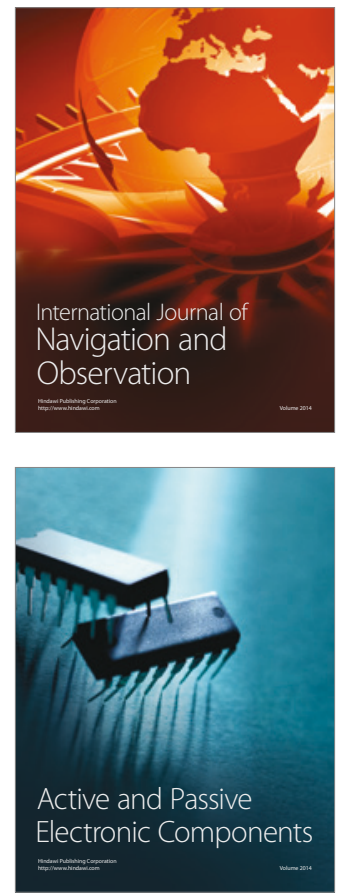
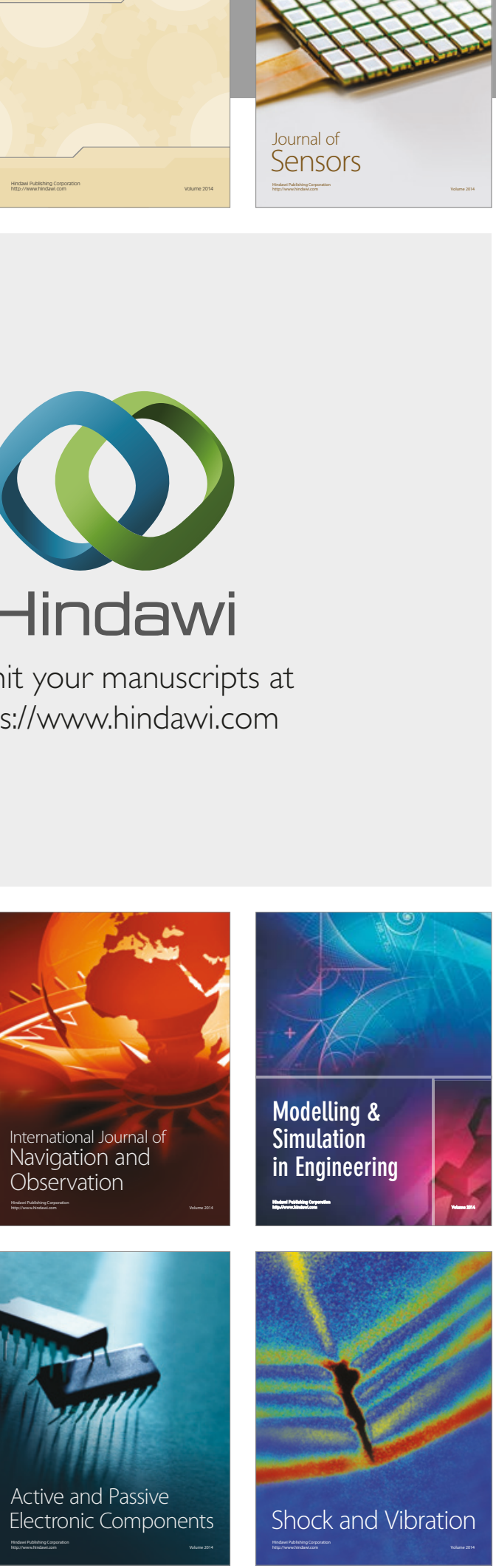
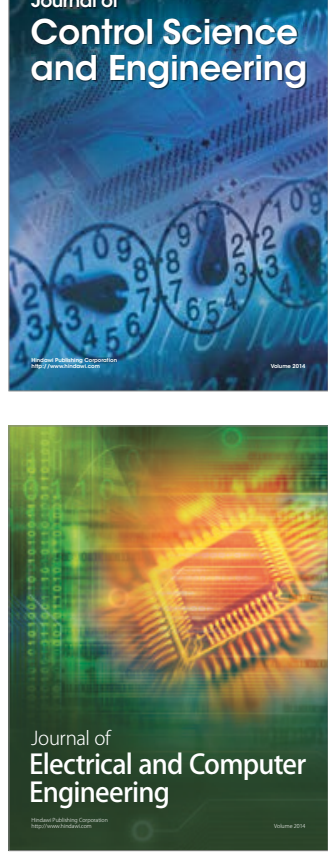

Distributed

Journal of

Control Science

and Engineering
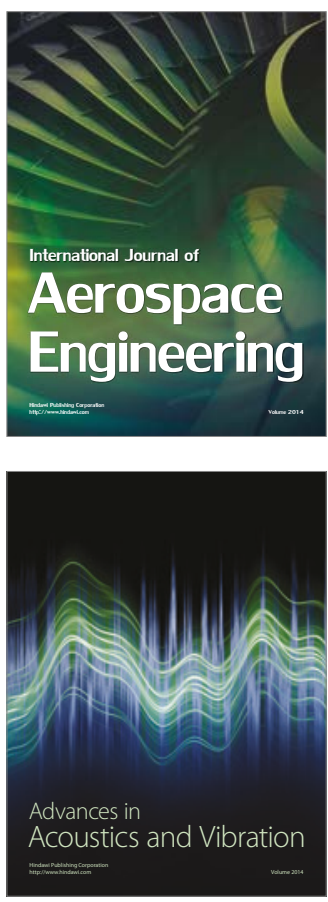

Sensor Networks 